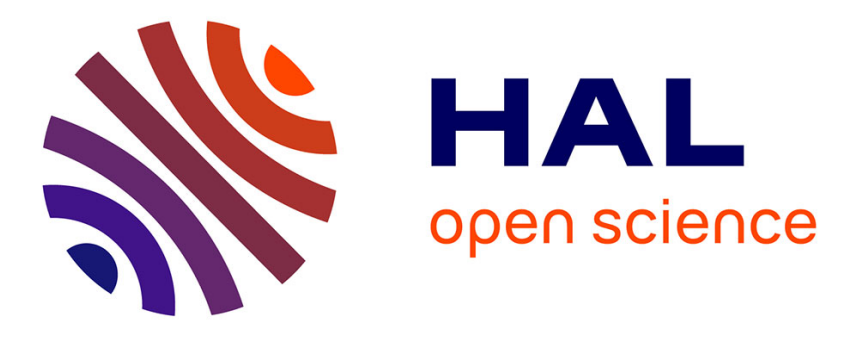

\title{
The effect of real-time auditory feedback on learning new characters
}

Jérémy Danna, Maureen Fontaine, Vietminh Paz-Villagrán, Charles Gondre, Etienne Thoret, Mitsuko Aramaki, Richard Kronland-Martinet, Sølvi Ystad, Jean-Luc Velay

\section{To cite this version:}

Jérémy Danna, Maureen Fontaine, Vietminh Paz-Villagrán, Charles Gondre, Etienne Thoret, et al.. The effect of real-time auditory feedback on learning new characters. Human Movement Science, 2015, 43, pp.216-228. 10.1016/j.humov.2014.12.002 . hal-01230184

\section{HAL Id: hal-01230184 \\ https://hal.science/hal-01230184}

Submitted on 24 Feb 2022

HAL is a multi-disciplinary open access archive for the deposit and dissemination of scientific research documents, whether they are published or not. The documents may come from teaching and research institutions in France or abroad, or from public or private research centers.
L'archive ouverte pluridisciplinaire HAL, est destinée au dépôt et à la diffusion de documents scientifiques de niveau recherche, publiés ou non, émanant des établissements d'enseignement et de recherche français ou étrangers, des laboratoires publics ou privés.

\section{(1) (1) $\$$}

Distributed under a Creative Commons Attribution - NonCommercial - NoDerivatives| 4.0 


\title{
The effect of real-time auditory feedback on learning new characters
}

\author{
Jérémy Danna ${ }^{\mathrm{a}, \mathrm{b}, *}$, Maureen Fontaine ${ }^{\mathrm{a}}$, Vietminh Paz-Villagrán ${ }^{\mathrm{a}}$, \\ Charles Gondre ${ }^{c}$, Etienne Thoret ${ }^{c}$, Mitsuko Aramaki ${ }^{c}$, \\ Richard Kronland-Martinet ${ }^{\mathrm{c}}$, Sølvi Ystad ${ }^{\mathrm{c}}$, Jean-Luc Velay ${ }^{\mathrm{a}}$ \\ a Laboratoire de Neurosciences Cognitives, UMR 7291, CNRS - Aix-Marseille Université, France \\ ${ }^{\mathrm{b}}$ Brain and Language Research Institute, CNRS - Aix-Marseille Université, France \\ ' Laboratoire de Mécanique et d'Acoustique, CNRS, UPR 7051, Aix-Marseille Université, Centrale Marseille, France
}

The present study investigated the effect of handwriting sonifica-tion on graphomotor learning. Thirty-two adults, distributed in two groups, learned four new characters with their non-dominant hand. The experimental design included a pre-test, a training ses-sion, and two post-tests, one just after the training sessions and another $24 \mathrm{~h}$ later. Two characters were learned with and two without real-time auditory feedback (FB). The first group first learned the two non-sonified characters and then the two sonified characters whereas the reverse order was adopted for the second group. Results revealed that auditory FB improved the speed and fluency of handwriting movements but reduced, in the short-term only, the spatial accuracy of the trace. Transforming kinematic variables into sounds allows the writer to perceive his/her move-ment in addition to the written trace and this might facilitate handwriting learning. However, there were no differential effects of auditory FB, neither longterm nor short-term for the subjects who first learned the characters with auditory FB. We hypothesize that the positive effect on the handwriting kinematics was trans-ferred to characters learned without FB. This transfer effect of the auditory FB is discussed in light of the Theory of Event Coding.

* Corresponding author at: Laboratoire de Neurosciences Cognitives, Pôle 3C, Case C, 3 Place Victor Hugo, 13331 Marseille Cedex 03, France. Tel.: +33413551168.

E-mail address: jeremy.danna@univ-amu.fr (J. Danna). 


\section{Introduction}

Sounds can naturally reveal phenomena that are external to our field of vision or that contain dynamic cues to which the eye is less sensitive (Fitch \& Kramer, 1994; McCabe \& Rangwalla, 1994). Consequently, they might form a powerful feedback (FB) medium for enriched motor training. Human movement sonification enables the exploration of new training methods in sports and motor learning, as well as the study of new therapeutic approaches in rehabilitation (Effenberg \& Mechling, 2005; Höner et al., 2011; Konttinen, Mononen, Viitasalo, \& Mets, 2004; Vogt, Pirrò, Kobenz, Höldrich, \& Eckel, 2009). The purpose of sonification is to enrich movement perception by transforming selected kinematic or dynamic movement parameters into congruent synthetic sounds. Sonification of movements seems to improve both their perception and reenactment (Effenberg, 2005; Young, Rodger, \& Craig, 2013). The relatively close neural connections between auditory and motor areas may explain these audio-motor interactions (Bengtsson et al., 2009; Haueisen \& Knöshe, 2001; Schmitz et al., 2013). Furthermore, a growing number of studies report that the audiovisual perception of sonified movement modulates the activity of the multisensory brain areas which then might lead to a more accurate representation of the movement (Bangert et al., 2006; Butler, James, \& James, 2011; Le Bel, Pineda, \& Sharma, 2009; Scheef et al., 2009; Schmitz et al., 2013). Finally, in addition to their informative characteristics, sounds can be playful and motivate learners (Schaffert, Barrass, \& Effenberg, 2009). Since handwriting learning requires daily training over several months, learner's motivation is an important component to take into account.

Yet, audition is not the sensory modality that is primarily associated with handwriting, which is a silent activity. A priori, proprioception would be more adapted to help writers to perceive the correct movement: Indeed, teachers do just this when they hold a child's hand and guide his/her movement (e.g. Bluteau, Coquillard, Payan, \& Gentaz, 2008; Teo, Burdet, \& Lim, 2002). However, there are limits to the use of supplementary proprioceptive guidance. First, its effectiveness has been addressed mostly on simple motor tasks (for a review, see Sigrist, Rauter, Riener, \& Wolf, 2013), but not on more complex tasks as handwriting. Secondly, proprioceptive guidance with force-feedback devices requires costly tools and complex implementation. In particular, this would first require recording an ideal trajectory that the writer would then have to reproduce and from which corrections could be made. Lastly, guiding the pen to the correct trajectory affects the action of the writer, tending her/him to some passiveness. Applying real-time auditory FB in handwriting is a more original approach, although first attempts were carried out a few decades ago in handwriting rehabilitation. For example, auditory FB was applied for the treatment of writer's cramp (Bindman \& Tibbetts, 1977; Reavley, 1975). The method consisted in transforming electromyography (EMG) recording into auditory biofeedback during relaxation and handwriting tasks. Although the method seemed attractive, Ince, Leon, and Christidis (1986) raised criticisms about these studies and reported some methodological difficulties. Notably, handwriting involves numerous small muscles which are not easily reachable with surface EMG. More recently, rather than applying auditory FB linked to the muscle activity, Baur, Fürholzer, Marquart, and Hermsdörfer (2009) applied auditory FB to the fingers' grip force on the pen for the treatment of writer's cramp. The auditory FB consisted in a continuous low-frequency tone when average grip force exceeded $5 \mathrm{~N}$ during handwriting. The tone frequency increased in four steps according to the grip force level and patients were instructed to perform the writing exercises in such a way that they heard a pleasant, low-frequency, tone. After seven hours of training, the grip force and the pressure applied by the pen on the paper decreased but the velocity and the fluency of their handwriting did not change significantly. These results were therefore encouraging for the rehabilitation of writer's cramp but not for handwriting movement improvement per se.

Plimmer, Reid, Blagojevic, Crossan, and Brewster (2011) tested a multimodal system based on auditory FB and haptic guidance for signature learning in blind children. The auditory FB consisted in sounds varying in stereo pan and pitch according to the $\mathrm{x}$ and $\mathrm{y}$ movement of the stylus, respectively. The haptic guidance was provided to the writer through a force-feedback haptic pen that reproduced the movement of the teacher's pen. While the authors concluded that the multisensory FB was efficient in helping the blind children write their signatures, this conclusion was not supported by a kinematic analysis. 
The first step was to check whether it was possible to associate sounds to handwriting variables in order to produce sound variations that, in turn, could enable the recognition of a graphical movement. This step was recently performed by Thoret, Aramaki, Kronland-Martinet, Velay, and Ystad (2014) who demonstrated that adult subjects were able to infer by ear what was drawn based, only, on the generated friction sounds. Their rationale was that, although handwriting is not really a noisy activity, the slight sounds generated by the pen friction on the paper can be heard in a silent environment. Consequently, these sounds can be processed, even unconsciously, and they could contribute to building a multimodal sensorimotor representation of handwriting. For a 'natural' sonification in which the mapping was quite spontaneous for the listeners: they chose sounds which afforded handwriting perception. They thus identified the acoustic cues that reflected the movements underlying a drawing action and they showed that a synthetic friction sound allows for the identification of simple graphic shapes from the corresponding velocity sonification. This study demonstrated that sounds can adequately give information about human movements if their dynamic acoustic characteristics are in accordance with the way the movements are performed. Therefore, to go a step further and following the same logic, it should be theoretically possible to perceive only by ear whether a handwriting movement is produced by a proficient or a poor writer.

In order to check the relevance of handwriting sonification for its evaluation, Danna, Paz-Villagrán, Gondre, et al. (2013) performed an experiment in which adult listeners had to grade various sonified handwriting only 'by ear', without seeing it. Three handwriting variables were sonified: 1 - Instantaneous speed, 2 - Fluency and 3 - Axial pen pressure on the tablet. Results showed that 'blind' listeners marked lower the handwriting of children with handwriting difficulties than that of proficient writers (children and adults). The conclusion was that the sonification of these three handwriting variables was sufficient to inform an external listener about the correctness of someone else's handwriting. In a second experiment, they tried to evaluate the effect of auditory FB in a dysgraphia rehabilitation protocol (Danna et al., 2014). The same real-time auditory FB was given to children with dysgraphia in order to help them correct their ongoing movement. Seven children practiced with such FB during four weekly sessions and did improve their handwriting speed and fluency. However, in this preliminary study, the absence of no-FB control conditions hindered any definitive conclusion as to whether this improvement resulted from the global training or from the auditory FB per se.

To our knowledge, no study has investigated the real-time auditory FB for handwriting learning, although applying sounds to help signature imitation has been attempted for evaluating the efficiency of a signature verification system (Plamondon, Yergeau, \& Brault, 1992). In that study two different sounds were produced according to the upward or downward displacement of the pen in the original signature. Imitators had therefore an idea of the pen lifts, the global direction (upward or downward), and the duration of each stroke to be copied. However, this method did not correspond to auditory FB because the imitators did not hear their own movement. Furthermore, this method did not help the imitators to deceive the verification system.

The aim of the present study was to evaluate the effect of real-time auditory FB on handwriting learning. Since we were not sure whether sonification has a positive effect on handwriting learning, we preferred to first conduct a study among adults, in whom a possible negative effect would have less impact than in children. In order to put the adults in a more difficult situation, comparable to that of a child who does not master handwriting, we asked them to write new characters with their nondominant hand.

\section{Method}

\subsection{Participants}

Thirty-two right-handed adults from the Aix-Marseille University (23 women, mean age 25:2 $\pm 3: 6$ ), distributed into two groups, volunteered for the experiment. All participants were French natives and did not have any knowledge about the Tamil script. They had normal or corrected-tonormal vision and normal audition. None of the participants presented any known neurological or attentional deficits, as determined by a detailed questionnaire prior to the experiment. The 
study was conducted in accordance with local norms and guidelines for the protection of human subjects.

\subsection{Task and procedure}

The task consisted in learning to write four new characters with the non-dominant hand (see Fig. 1). The participants were required to write the characters on a sheet of paper (A4 format: $21.0 \times 29.7 \mathrm{~cm}$ ) affixed to a graphic tablet (Wacom, Intuos3 A4, sampling frequency $200 \mathrm{~Hz}$ ) using an ink pen. The character was presented at the top of the sheet. A square $(4.0 \times 4.0 \mathrm{~cm})$ was drawn on the bottom side of the sheet for each character and each repetition.

The experimental design included a short familiarization with the FB, a pre-test, a training session, and two post-tests. The first post-test was performed just after the training session of each character, and the second the following day (see Fig. 2). The pre-test and the two post-tests were exactly the

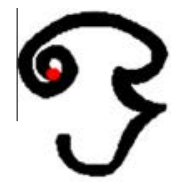

A

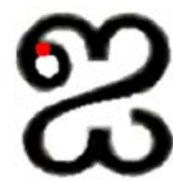

B

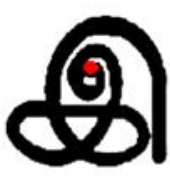

C

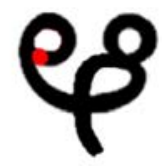

D

Fig. 1. The four learned characters were extracted from the Tamil script. Character D was slightly modified in order to be drawn without lifting the pen. A red point has been added to indicate to participants' the starting point. (For interpretation of the references to color in this figure legend, the reader is referred to the web version of this article.)

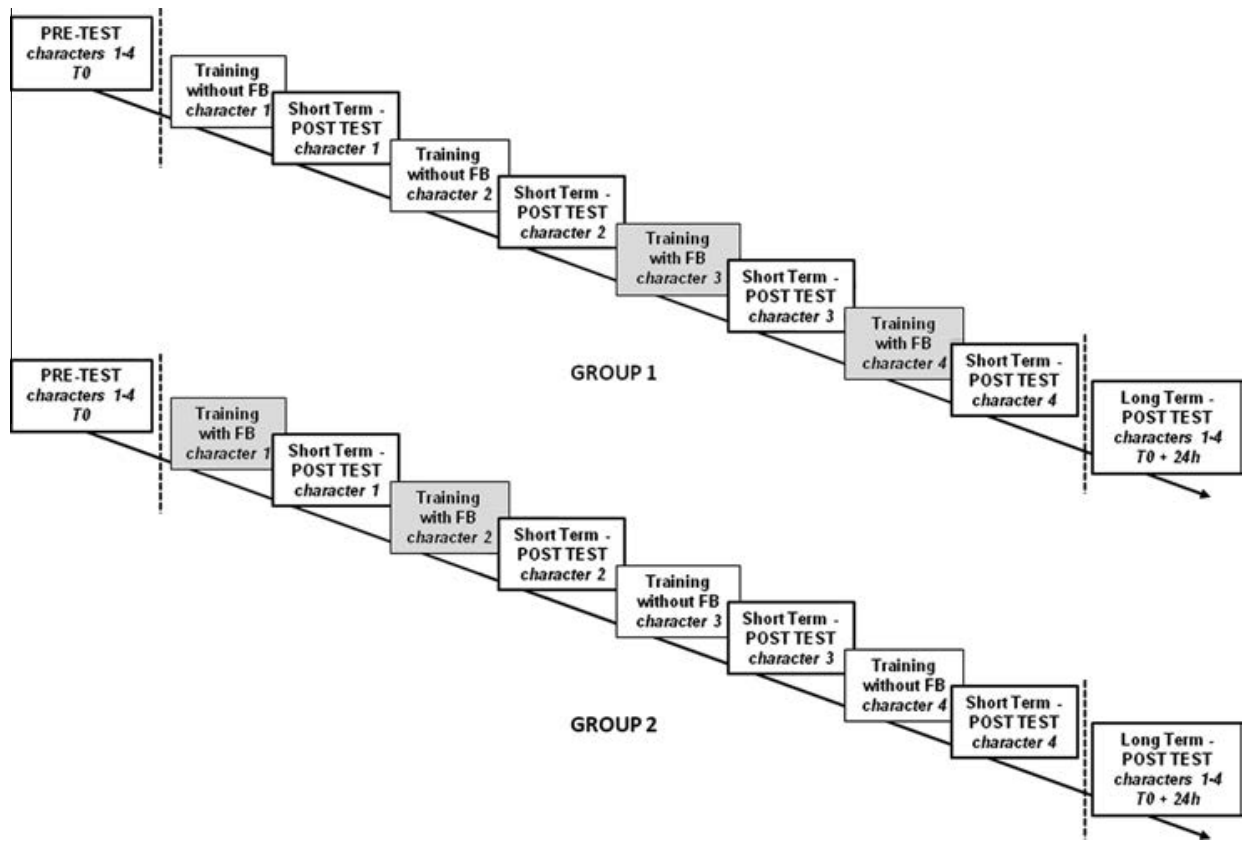

Fig. 2. Experimental design. Group 1 learned the two characters with the auditory feedback (in gray) after the two characters learned without auditory feedback (in white) whereas Group 2 learned the characters with the auditory feedback before learning characters without auditory feedback. 
same in order to allow for comparison. The participants wrote each character one time without auditory FB. During the training session, participants wrote each character 16 times, with real-time auditory FB for two of them.

The difference between the two groups concerned the order of characters learned with and without the auditory FB. Group 1 learned the two characters with auditory FB after the two characters learned without auditory FB whereas Group 2 learned the characters with the auditory FB before the learning of characters without auditory FB (see Fig. 2). The distribution and the order of the characters (A-D) were pseudo-counterbalanced across the training session (1-4) for the participants of each group. Consequently, the four characters were equally learned with and without auditory FB for each group.

\subsection{Movement sonification}

In the present study, the purpose of sonification is to use synthetic sounds that provide support for information processing (Barrass \& Kramer, 1999). For that, metaphoric sounds, i.e. sounds evoking a natural action on an object, were used (Aramaki \& Kronland-Martinet, 2006; Conan et al., 2013; Thoret, Aramaki, Gondre, Kronland-Martinet, \& Ystad, 2013). Indeed, the effectiveness of auditory FB strongly depends on the intuitive and correct interpretation of the applied mapping functions and metaphors (Castiello, Giordano, Begliomini, Ansuini, \& Grassi, 2010).

We thus associated a rubbing sound to a correct handwriting. This sound was close to the sound generated by writing with a chalk on a blackboard. When handwriting was too slow, the rubbing sound changed into squeaking sound. This second sonification strategy results from the metaphor of the squeaking of a door which naturally leads the writers to increase their movement speed to avoid this unpleasant noise. Finally, additional impact sounds, recalling cracking sounds appeared when the handwriting was jerky. These additional discrete sounds reinforced the metaphor of lack of fluency in producing a continuous action (e.g. chalk that breaks while writing or vinyl that cracks...).

To present and describe the method of movement sonification, a video is available with the electronic version of the manuscript. This video presents a prototypical improvement of performance with training. From the first to the eighth trial, a progressive change of sounds can be perceived, from squeaking to rubbing sounds with improvement of speed production, and a decrease of impact sounds with improvement of movement fluency.

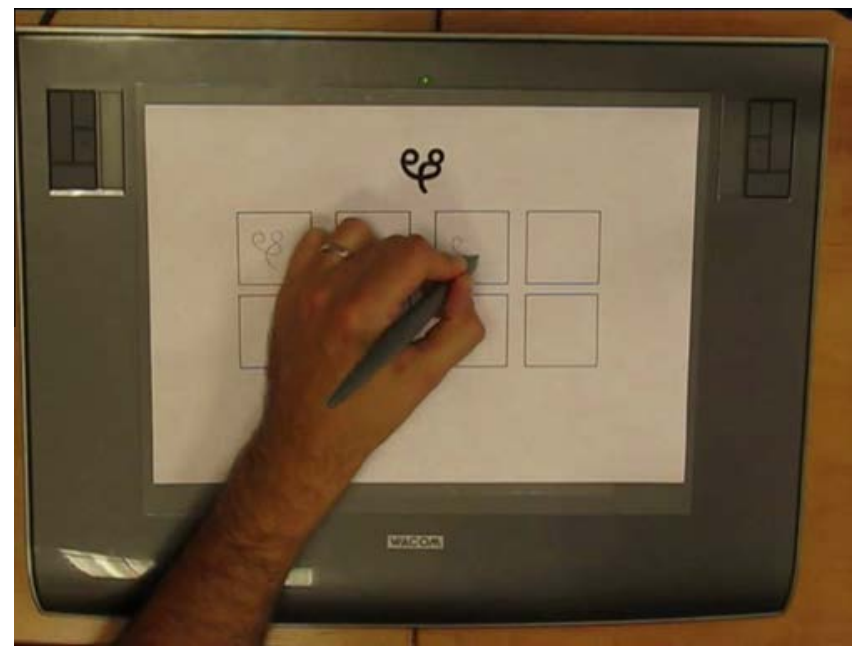

Supplementary video 1 
The synthesis model was based on a source-resonator model. This model results from a sound synthesis action/object paradigm in which the sound is defined as the result of an action on an object. In this paradigm, the object's properties are separated from the interactions it is subjected to. This model is an approximation of physical modeling: it stands that in an interaction, the physical exciter is decoupled from the resonator. In the case of continuous sounds, the interaction (source) can be represented by an adequate excitation signal while the object's modes (resonator) can be represented by an adequate resonant filter bank (Conan et al., 2013). The source was modeled in two different manners, depending on the gesture velocity. For high velocities, rubbing sounds corresponding to noisy sounds were produced by lowpass filtering a noise with a cutoff frequency linked to the velocity of the gesture (for more details, see Conan et al., 2013). For velocities considered too slow, squeaking sounds based on non-linear (stick-slip) friction behavior were generated (for more details, see Thoret et al., 2013). The synthesis model enabled sudden transitions between squeaking sounds and rubbing sounds. In the present study, handwriting was considered too slow when the instantaneous tangential velocity was lower than $1.5 \mathrm{~cm} \mathrm{~s}^{-1}$. Consequently, the transition between the friction sound and the squeaking sound was made at this threshold.

Finally, the impact sounds were synthesized by the synthesizer developed by Aramaki and colleagues (for more details, see Aramaki, Besson, Kronland-Martinet, \& Ystad, 2011; Aramaki, Gondre, Kronland-Martinet, Voinier, \& Ystad, 2010). These sounds were produced when the time between two velocity peaks was less than $40 \mathrm{~ms}$ and when the velocity difference between these two peaks was less than $1.0 \mathrm{~cm} \mathrm{~s}^{-1}$. These thresholds were determined empirically and validated from the high correlation between the number of supra-threshold velocity peaks determined by this real-time method and the number of supra-threshold velocity peaks determined by the Signal-toNoise velocity peak difference (SNvpd), index of movement fluency (Danna, Paz-Villagrán, \& Velay, 2013).

\subsection{Data analysis}

Two different types of variables were computed: kinematic variables on handwriting movement and spatial variables on the written trace.

\subsubsection{Kinematic variables}

The mean velocity and the SNvpd were computed. The mean velocity corresponds to the mean of tangential velocity from the time the pen is in contact with the tablet until it lifts, when the character is completed. The SNvpd is the difference between the number of velocity peaks after filtering the tangential velocity with a frequency cutoff ( $\mathrm{fc}$ ) of $10 \mathrm{~Hz}$ and the number of velocity peaks after filtering the tangential velocity with a fc of $5 \mathrm{~Hz}$ (Danna, Paz-Villagrán, \& Velay, 2013).

\subsubsection{Spatial variables}

The trace length and the Dynamic Time Warping (DTW) distance were computed. Trace length corresponds to the total trajectory followed by the pen to write the character. DTW is the measurement of the Euclidean distance between the character written by participants and a character prototype considered as a reference. Historically, DTW was a technique introduced for pattern recognition in handwriting (Di Brina, Niels, Overvelde, Levi, \& Hulstijn, 2008; Niels, Vuurpijl, \& Schomaker, 2007). Technically, DTW corresponds to a point-to-point comparison between two characters in which both spatial and temporal information is available. The DTW distance is computed as the average Euclidean distance between all pairs of matching points (for more details about criteria used for matching, see Niels et al., 2007). In the present experiment, the four character prototypes were realized by a proficient adult who practiced to write each character with the dominant hand and with the aid of the model until the perfect shape was attained. The series of $(x, y)$ coordinates corresponding to the shape of each character were then filtered with a 4th order low-pass Butterworth filter with a fc of $5 \mathrm{~Hz}$. 
These four characters could be considered as 'ideal' characters and the greater the dissemblance between them and the character drawn by a subject, the greater the DTW distance. Here we took the inverse of DTW distance which was considered as an index of spatial accuracy: The better the character matched with the reference, the higher the score.

\subsection{Statistical analysis}

The four variables were averaged separately for the two characters written during the training phase with auditory FB and for the two written without auditory FB. Then, they were submitted to a three-way analysis of variance (ANOVA) with three 'Learning' conditions (pre-test, short term post-test, and long term post-test) and two 'Feedback' conditions (with or without auditory FB) as repeated measures, and with the two 'Presentation Order' (characters learned with auditory FB before or after the characters learned without auditory FB) as group factor. All significance thresholds were set to $p<.05$. For all ANOVAs, Fisher's LSD post hoc tests with Bonferroni's correction were applied when necessary.

\section{Results}

\subsection{Movement velocity}

The ANOVA revealed a main effect of Learning $(F(2,60)=63.27, p<.001)$ : Post-hoc tests showed a significant difference between all three test phases $(p<.001)$. The Learning by Feedback interaction was significant $(F(2,60)=3.98, p<.05)$ : Post-hoc tests revealed that the characters learned with the auditory FB were produced with higher velocity than the characters learned without the auditory FB in the short-term post-test $(p<.01)$ but not in the long-term post-test $(p=.22)$. Finally, the Learning by Feedback by Order three-way interaction was also significant $(F(2,60)=8.91, p<.001$; Fig. 3$)$ : Post-hoc tests revealed that the characters learned with the auditory FB were produced with higher velocity than the characters learned without the auditory FB in the short-term post-test (TO) for Group 1 only, i.e. when the characters with the auditory FB were learned after those without the auditory FB $(p<.0001)$.

In conclusion, when the participants began the training sessions without auditory FB (Group 1), the characters learned with FB were written faster than those learned without FB in the short-term post-test. When the participants began the training sessions with the auditory FB (Group 2), the characters learned without FB were written at the same velocity than those learned with the auditory FB.

\subsection{Movement fluency}

The ANOVA revealed a main effect of Learning $(F(2,60)=21.97, p<.001)$ : Post-hoc tests showed a significant difference between all three test phases $(p<.001)$. The Learning by Feedback interaction was significant $(F(2,60)=4.06, p<.05)$ : Post-hoc tests revealed that the characters learned with the auditory FB were produced more fluently than those learned without the auditory FB in the short-term $(p<.05)$ but not the long-term post-test $(p=.65)$. Finally, the Learning by Feedback by Order three-way interaction was also significant $(F(2,60)=4.57, p<.05$; see Fig. 4$)$ : Posthoc tests revealed that the characters learned with the auditory FB were produced more fluently than the characters learned without the auditory FB in the short-term post-test (TO) for Group 1 only, i.e. when the characters with the auditory FB were learned after those without the auditory FB $(p<.01)$.

In conclusion, when participants began the training sessions without auditory FB (Group 1), the characters learned with FB were written more fluently than those learned without it in the short-term post-test. When the participants began the training sessions with the auditory FB (Group 2), the 

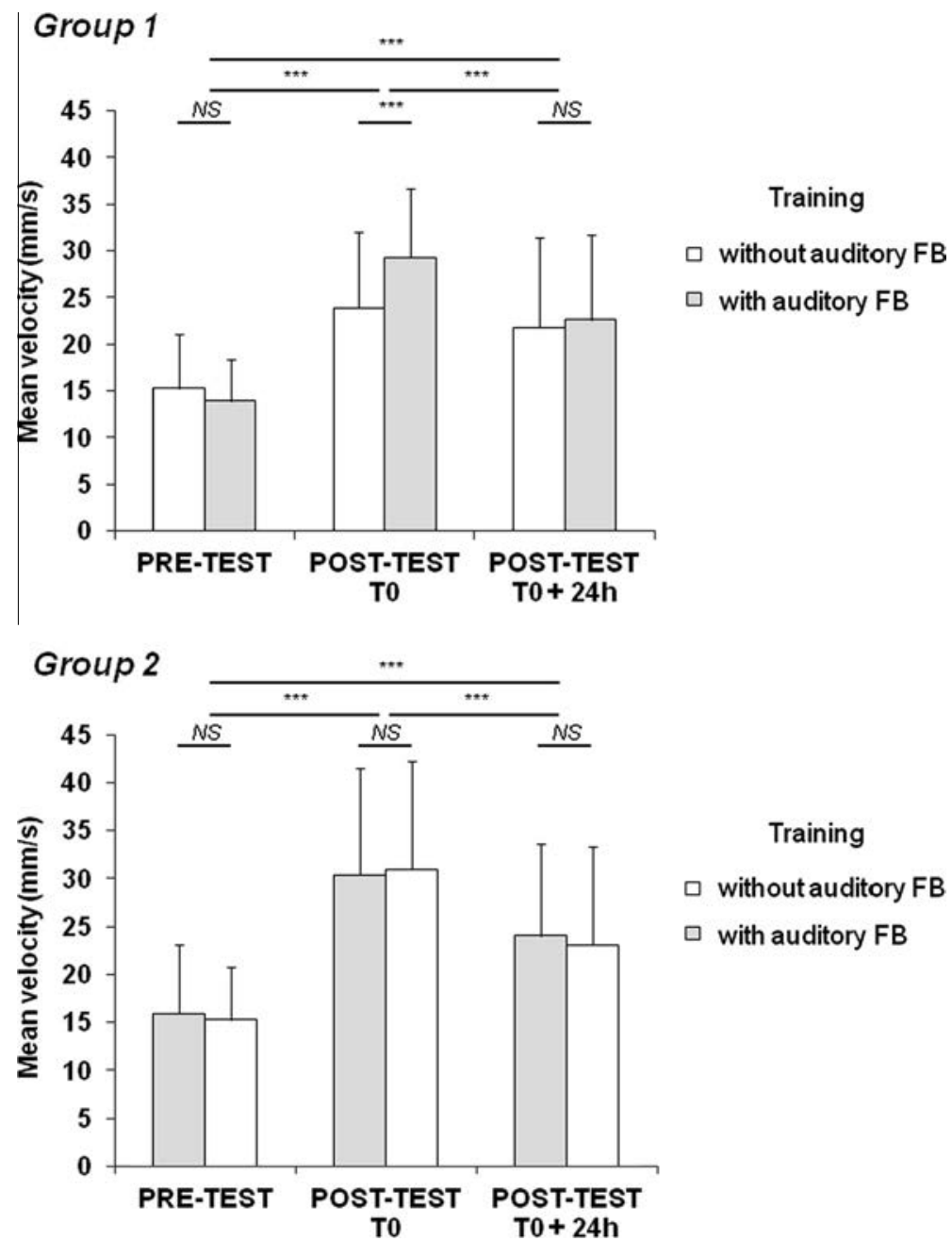

Fig. 3. Mean velocity in the pre-test, the short term post-test, and the long term post-test of characters learned with or without auditory feedback during the training phase. Group 1 first learned characters without auditory FB whereas Group 2 first learned characters with auditory FB. Error bars correspond to inter-participants SD. ${ }^{* * *} p<.001$.

characters learned without FB were written with the same fluency than those learned with the supplementary FB.

\subsection{Trace length}

The ANOVA revealed only a marginal effect of Learning $(F(2,60)=2.98, p=.06)$ : Post-hoc tests showed that the mean length of characters written during the long-term post-test tended to be longer than that of characters written during the pre-test $(108 \mathrm{~mm}$ in the pre-test and $113 \mathrm{~mm}$ in the long term post-test; $p=.06$ ). Adding auditory FB did not change the length of the characters in the posttests. 

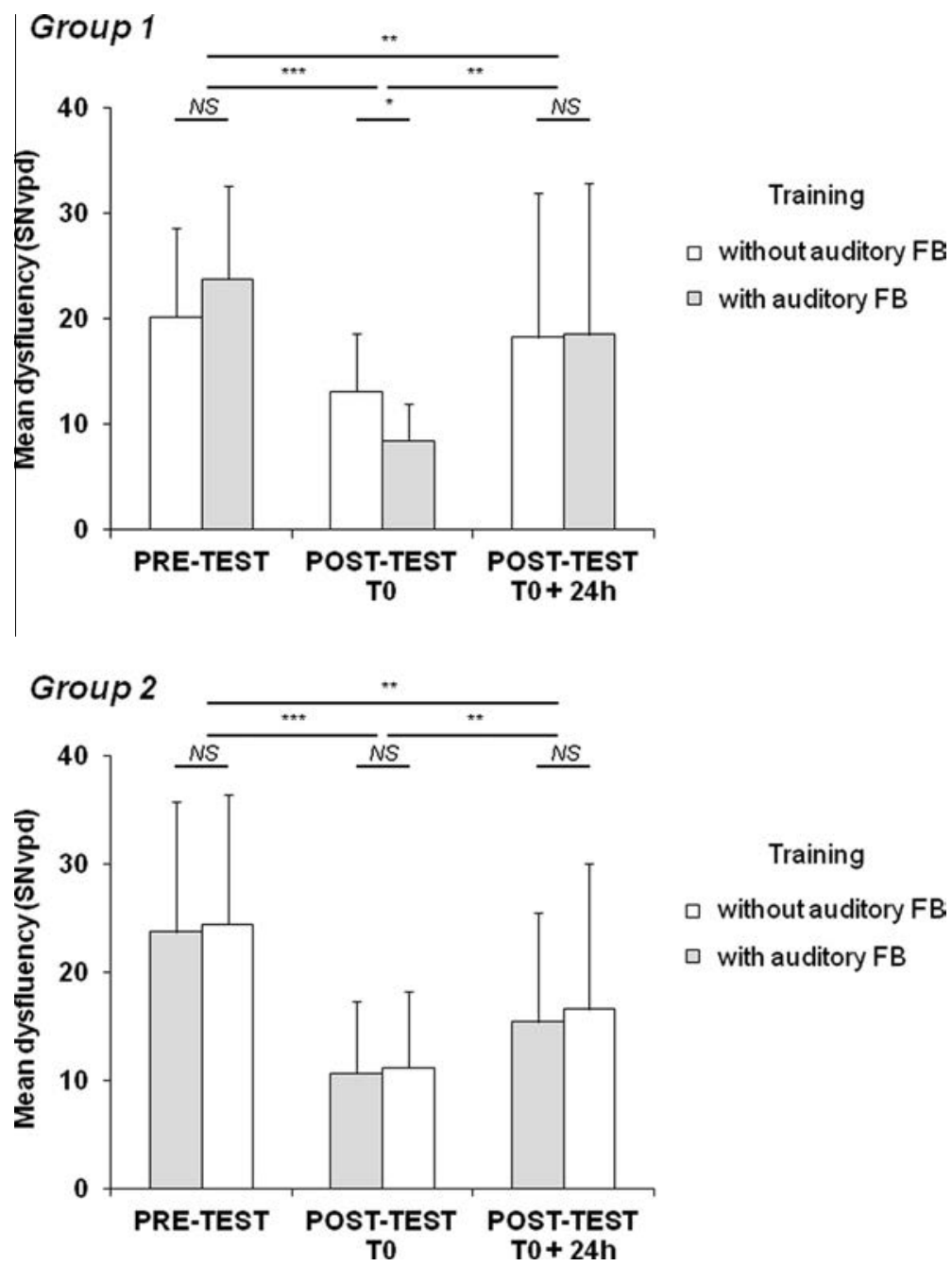

Fig. 4. Mean dysfluency in the pre-test, the short term post-test, and the long term post-test for characters learned with or without auditory feedback during the training phase. Group 1 first learned characters without auditory FB whereas Group 2 first learned characters with auditory FB. Error bars correspond to inter-participants SD. ${ }^{*} p<.05 ;{ }^{* *} p<.01 ;{ }^{* * *} p<.001$.

\subsection{Spatial accuracy}

The ANOVA revealed a main effect of Learning $(F(2,60)=9.24, p<.001)$ : Post-hoc tests of the Learning effect showed that the spatial accuracy in the short-term post-test was lower than the spatial accuracy in the pre-test and the long term post-test $(p<.01)$ which did not differ between them. The ANOVA also revealed a Learning by Feedback interaction $(F(2,60)=6.28, p<.01$; see Fig. 5$)$ : Post-hoc tests revealed that the characters learned with the auditory FB were produced with a lower accuracy than the characters learned without FB in the short term post-test (TO) only, whatever the order of presentation of the training sessions $(p<.05)$. The Learning by Feedback by Order double interaction was not significant $(p=.87)$. Consequently, both groups were merged in Fig. 5 . 


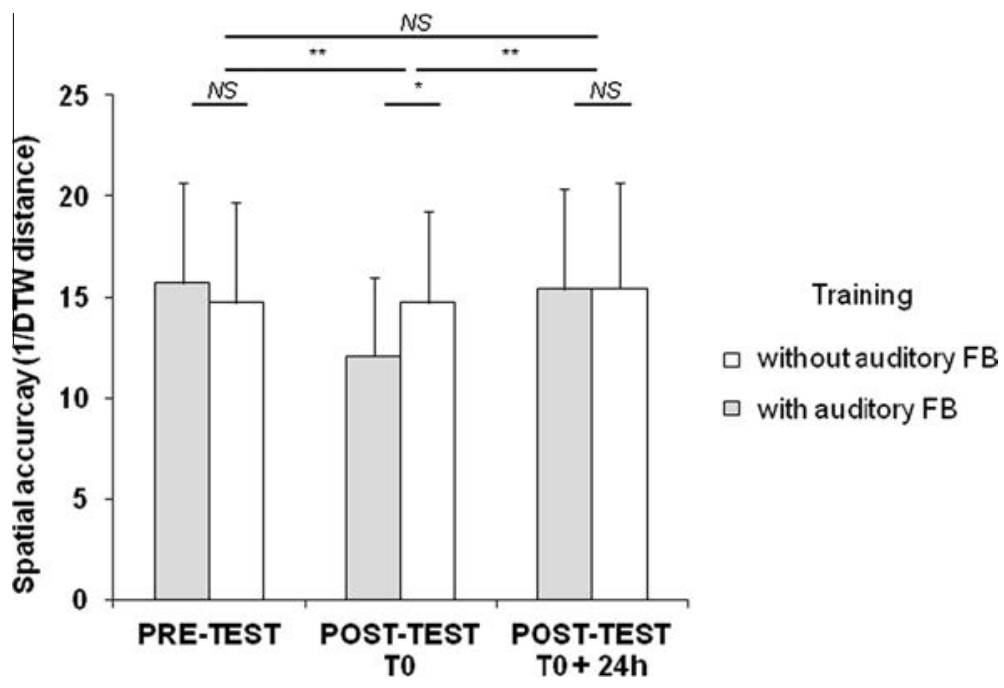

Fig. 5. Mean spatial accuracy in the pre-test, the short term post-test, and the long term post-test for characters learned with and without auditory feedback during the training phase. Both groups are merged. Error bars correspond to inter-participants SD. ${ }^{*} p<.05 ;{ }^{* *} p<.01$.

\section{Discussion}

The aim of the present study was to evaluate the effect of handwriting movement sonification on new character learning. Two groups of right-handed adults learned four unknown characters with their non-dominant hand, with or without real-time auditory FB impacting on the velocity and the fluency of their movement. Among the four characters, two were learned with the aid of auditory FB and two were learned without. Half of the participants (Group 1) first learned the two characters without the auditory FB and the other half (Group 2) first learned the two characters with the FB.

When comparing the performance at the short-term post-test, two main results emerged. First, applying real-time auditory FB when learning to write new characters improved the kinematics of the handwriting movements, though at the cost of a lower spatial accuracy. It is likely that increasing information on the kinematics during learning made the writers pay more attention to these characteristics of the movement and less to the spatial features of the written trace. In handwriting control, spatial information is mainly provided by vision (Smyth \& Silvers, 1987) whereas kinematic information is mainly provided by proprioception (Hepp-Reymond, Charakov, Schulte-Mönting, Huethe, \& Kristeva, 2009). Adding auditory kinematic signals might have decreased the visual control of the trace and thus may have reduced the spatial precision. At the same time, reducing the time-consuming visual control could have resulted in an improvement of the velocity and the fluency of the movement. This is in line with the study of Chartrel and Vinter (2008) showing that, in handwriting learning among children, imposing temporal constraints forced the reduction of visual control and allowed for the programming of longer movements, resulting in improved fluency. In a similar vein, Portier and van Galen (1992) demonstrated that real-time supplementary visual FB induced most explicitly an on-line visual control which increased the dysfluency of handwriting.

Secondly, in the short-term test, the characters learned without auditory FB were written with a slower velocity and fluency than the characters having benefited from the FB. This observation was only valid for Group 1 in which the FB was applied for the last two characters. Conversely, when the auditory FB was applied for the first two characters (Group 2), the performance of characters learned with and without auditory FB were quite similar. In other words, the FB effect on the handwriting kinematics was transferred to characters learned without FB, provided that they were learned after those having been associated to the auditory FB. Interestingly, this transfer seems to concern the 
kinematic features of handwriting movement only (velocity and fluency), since the spatial accuracy remained identical for characters learned without auditory FB, whatever the order of presentation of the FB.

This transfer of the auditory FB to the production of the other characters in no-FB condition can be discussed in light of the Theory of Event Coding (Hommel, Müsseler, Aschersleben, \& Prinz, 2001; Prinz, 1997), which considers cognitive representations as a structural coupling between perception and action. In this view, multisensory signals (visual, proprioceptive and auditory) would be integrated during the learning with the auditory FB, to provide a unified percept of the "writing event". This multimodal representation would be then reactivated when new characters have to be learned and the internalized sounds associated to the movement would be given even if they are not physically supplied. This hypothesis is supported by studies showing that executing silent finger movements on a piano keyboard elicited stronger activation of auditory-sensory areas with expertise (Bangert, Häusler, \& Altenmüller, 2001) or training (Bangert \& Altenmüller, 2003; Engel et al., 2012). The combined auditory FB and motor training on the piano resulted in the coactivation of cortical auditory and sensorimotor hand regions in either pure auditory or silent motor tasks in such a way that silent dexterity drills produce "audible tones inside the head" (Bangert et al., 2001, p. 425).

When the subjects wrote the four characters $24 \mathrm{~h}$ after the end of the training, they still wrote faster and more fluently than before training but without a decrease in their spatial accuracy. Furthermore, there was no longer a difference between the characters learned with or without the auditory FB. The absence of a long-term FB effect could result from two different but not mutually exclusive causes. First, it could result from the limited number of writing repetitions during the training session (16 of each character). If this explanation holds, increasing the number of trials in the training session, or the number of training sessions, would induce more long-lasting effects of the auditory FB. Secondly, the auditory FB effect transferred towards the characters learned without it.

Regarding the dependence on the external FB, the "guidance hypothesis" states that permanent FB during acquisition leads to a dependency on the FB (Schmidt, Young, Swinnen, \& Shapiro, 1989). However, two reasons can limit such a hypothetical effect in the present case. First, handwriting can be considered as one of the most complex tasks, since it involves a sophisticated coordination of many muscles and joints (Van Emmerick \& Newell, 1989). Sigrist et al. (2013) reported that the more complex the task, the more the trainee can benefit from concurrent FB. In an early learning phase of complex tasks, concurrent FB would attract an external focus of attention (Wulf, 2013). Secondly, Ronsse et al. (2011) demonstrated that, in a motor learning task, learners are less dependent on auditory than on visually-augmented FB. Indeed, only auditory FB resulting from sonification can be internalized and recalled in no-feedback conditions. Knowing the transfer effect of auditory FB, it is possible to manipulate the schedule of FB presentation such as reducing the frequency at which the FB is presented (Wulf \& Schmidt, 1989) with a strategy adapted for children who use FB differently than adults (Sullivan, Kantak, \& Burtner, 2008).

In conclusion, the present experiment showed that sonifying handwriting during the learning of unknown characters improved the fluency and speed of their production, despite a slight reduction of their short-term spatial accuracy. These results are promising since they validate the use of sounds for informing about handwriting kinematics. Transforming kinematic variables into sounds amounts to translating proprioceptive signals into exteroceptive signals, thus revealing hidden characteristics of the handwriting movement to the writer. This sonification method seems to be efficient, at least in proficient adults. Of course, the main purpose of handwriting sonification is the learning and the rehabilitation of handwriting in children. This sonification method has been tested with children with dysgraphia and has appeared to be efficient (Danna et al., 2014). However, other tests must be conducted in order to validate it. Particular attention will be paid to the sound design. Indeed, arbitrarily designed displays may constrain motor learning by reduced motivation distraction, or misinterpretation. With this in mind, we envisage using an adaptation of the Questionnaire for User Interface Satisfaction (Chin, Diehl, \& Norman, 1988) for children to optimize their motivation to write. Another possibility involving the application of more musical sounds to provide more pleasant FB will also be investigated. This possibility has already been successfully applied in a synchronization task (Varni et al., 2012) and the idea of producing music with writing seems promising. 


\section{Acknowledgments}

This work, carried out within the Labex BLRI (ANR-11-LABX-0036), has benefited from support from the French Government, managed by the French National Agency for Research (ANR), under the project title Investments of the Future A*MIDEX (ANR-11-IDEX-0001-02) and under the project METASON CONTINT (ANR-10-CORD-0003). The authors acknowledge Jessica Hackett for English revision.

\section{References}

Aramaki, M., \& Kronland-Martinet, R. (2006). Analysis-synthesis of impact sounds by real-time dynamic filtering. IEEE Transactions on Audio Speech, and Language Processing, 14(2), 695-705.

Aramaki, M., Besson, M., Kronland-Martinet, R., \& Ystad, S. (2011). Controlling the perceived material in an impact sound synthesizer. IEEE Transactions on Audio Speech, and Language Processing, 19(2), 301-314.

Aramaki, M., Gondre, C., Kronland-Martinet, R., Voinier, T., \& Ystad, S. (2010). Imagine the sounds: An intuitive control of an impact sound synthesizer. Auditory Display, Lecture Notes in Computer Science, 5954, 408-421.

Bangert, M., \& Altenmüller, E. (2003). Mapping perception to action in piano practice: A longitudinal DC-EEG study. BMC Neuroscience, 4, 26.

Bangert, M., Häusler, U., \& Altenmüller, E. (2001). On practice: How the brain connects piano keys and piano sounds. Annals of the New York Academy of Sciences, 930, 425-428.

Bangert, M., Peschel, T., Schlaug, G., Rotte, M., Drescher, D., Hinrichs, H., et al (2006). Shared networks for auditory and motor processing in professional pianists: Evidence from fMRI conjunction. Neuroimage, 30(3), 917-926.

Barrass, S., \& Kramer, G. (1999). Using sonificiation. Multimedia systems, 7, 23-31.

Baur, B., Fürholzer, W., Marquart, C., \& Hermsdörfer, J. (2009). Auditory grip force feedback in the treatment of Writer's Cramp. Journal of Hand Therapy, 22(2), 163-170.

Bengtsson, S. L., Ullén, F., Ehrsson, H. H., Hashimoto, T., Kito, T., Naito, E., et al (2009). Listening to rhythms activates motor and premotor cortices. Cortex, 45, 62-71.

Bindman, E., \& Tibbetts, R. W. (1977). Writer's cramp - A rational approach to treatment? The British Journal of Psychiatry, 131, $143-148$

Butler, A. J., James, T. W., \& James, K. H. (2011). Enhanced multisensory integration and motor reactivation after active motor learning of audiovisual associations. Journal of Cognitive Neuroscience, 23(11), 3515-3528.

Bluteau, J., Coquillard, S., Payan, Y., \& Gentaz, E. (2008). Haptic guidance improves the visuo-manual tracking of trajectories. PLoS One, 3(3), e1775.

Castiello, U., Giordano, B. L., Begliomini, C., Ansuini, C., \& Grassi, M. (2010). When ears drive hands: The influence of contact sound on reaching to grasp. PLoS One, 5(8), e12240. http://dx.doi.org/10.1371/journal.pone.0012240.

Chartrel, E., \& Vinter, A. (2008). The impact of spatio-temporal constraints on cursive letter handwriting in children. Learning and Instruction, 18, 537-547.

Chin, J., Diehl, V., \& Norman, K. (1988). Development of an instrument measuring user satisfaction of the human-computer interface. In Proceedings of ACM CHI '88. New York, USA: ACM Press.

Conan, S., Thoret, E., Aramaki, M., Derrien, O., Gondre, C., Kronland-Martinet, R., et al (2013). Navigating in a space of synthesized interactions-sounds: Rubbing, scratching and rolling. In Proceedings of the 16th conference on digital audio effects (DAFx). Ireland: Maynooth.

Danna, J., Paz-Villagrán, V., Gondre, C., Aramaki, M., Kronland-Martinet, R., Ystad, S., et al (2013). Handwriting sonification for the diagnosis of dysgraphia. In M. Nakagawa, M. Liwicki, \& B. Zhu (Eds.), Recent progress in graphonomics: Learn from the past - Proceedings of the 16th conference of the international graphonomics society (pp. 123-126). Tokyo, Japan: Tokyo University of Agriculture and Technology Press.

Danna, J., Paz-Villagrán, V., \& Velay, J.-L. (2013). Signal-to-Noise velocity peaks difference: A new method for evaluating the handwriting movement fluency in children with dysgraphia. Research in Developmental Disabilities, 34(12), 4375-4384.

Danna, J., Paz-Villagrán, V., Capel, A., Petroz, C., Gondre, C., Pinto, S., et al (2014). Handwriting movement sonification for the diagnosis and the rehabilitation of graphomotor disorders. In M. Aramaki, O. Derrien, R. Kronland-Martinet, \& S. Ystad (Eds.), Sound, Music \& Motion. Berlin Heidelberg: Springer [LNCS 8905].

Di Brina, C., Niels, R., Overvelde, A., Levi, G., \& Hulstijn, W. (2008). Dynamic time warping: A new method in the study of poor handwriting. Human Movement Science, 27(2), 242-255.

Effenberg, A. O., \& Mechling, H. (2005). Movement-sonification: A new approach in motor control and learning. Journal of Sport E' Exercise Psychology, 27, 58.

Effenberg, A. O. (2005). Movement sonification: Effects on perception and action. IEEE Multimedia, 12(2), 53-59.

Engel, A., Bangert, M., Horbank, D., Hijmans, B. S., Wilkens, K., Keller, P. E., et al (2012). Learning piano melodies in visuo-motor or audio-motor training conditions and the neural correlates of their cross-modal transfer. NeuroImage, 63, 966-978. 
Fitch, W. T., \& Kramer, G. (1994). Sonifying the body electric: Superiority of an auditory over a visual display in a complex, multivariate system. In G. Kramer (Ed.), Auditory display: Sonification, audification and auditory interfaces (pp. 307-325). Reading MA: Addison-Wesley.

Haueisen, J., \& Knöshe, T. R. (2001). Involuntary motor activity in pianists evoked by music perception. Journal of Cognitive Neuroscience, 13, 786-792.

Hepp-Reymond, M. C., Charakov, V., Schulte-Mönting, J., Huethe, F., \& Kristeva, R. (2009). Role of proprioception and vision in handwriting. Brain Research Bulletin, 79, 365-370.

Hommel, B., Müsseler, J., Aschersleben, G., \& Prinz, W. (2001). The theory of event coding (TEC): A framework for perception and action planning. Behavioral and Brain Sciences, 24(5), 849-878.

Höner, O., Hunt, A., Pauletto, S., Röber, N., Hermann, T., \& Effenberg, A. O. (2011). Aiding movement with sonification in "Exercice, Play and Sport". In T. Hermann, A. Hunt, \& J. G. Neuhoff (Eds.), The sonification handbook (pp. 525-553). Berlin, Germany: Logos Publishing House.

Ince, L. P., Leon, M. S., \& Christidis, D. (1986). EMG biofeedback for handwriting disabilities: A critical examination of the littérature. Journal of Behavioral Therapy and Experimental Psychiatry, 17(2), 95-100.

Konttinen, N., Mononen, K., Viitasalo, J., \& Mets, T. (2004). The effects of augmented auditory feedback on psychomotor skill learning in precision shooting. Journal of Sport E' Exercise Psychology, 26, 306-316.

Le Bel, R. M., Pineda, J. A., \& Sharma, A. (2009). Motor-auditory-visual integration: The role of the human mirror neuron system in communication and communication disorders. Journal of Communication Disorders, 42, 299-304.

McCabe, K., \& Rangwalla, A. (1994). Auditory display of computational fluid dynamics Data. In G. Kramer (Ed.), Auditory display: Sonification, audification and auditory interfaces (pp. 327-340). Reading MA: Addison-Wesley.

Niels, R., Vuurpijl, L., \& Schomaker, L. (2007). Automatic allograph matching in forensic writer identification. International Journal of Pattern Recognition and Artificial Intelligence, 21(1), 61-81.

Plamondon, R., Yergeau, P., \& Brault, J. J. (1992). A multi-level signature verification system. In S. Impedovo \& J. C. Simon (Eds.), From pixels to features III: Frontiers in handwriting recognition (pp. 363-370). Amsterdam: Elsevier.

Plimmer, B., Reid, P., Blagojevic, R., Crossan, A., \& Brewster, S. (2011). Signing on the tactile line: A multimodal system for teaching handwriting to blind children. ACM Transactions on Computer Human Interaction, 18(3), 1-29.

Portier, S. J., \& van Galen, G. P. (1992). Immediate vs. postponed visual feedback in practicing a handwriting task. Human Movement Science, 11, 563-592.

Prinz, W. (1997). Perception and action planning. European Journal of Cognitive Psychology, 9(2), 129-154.

Reavley, W. (1975). The use of biofeedback in the treatment of writer's cramp. Journal of Behavioral Therapy and Experimental Psychiatry, 6, 335-338.

Ronsse, R., Puttemans, V., Coxon, J. P., Goble, D. J., Wagemans, J., Wenderoth, N., et al (2011). Motor learning with augmented feedback: Modality-dependent behavioral and neural consequences. Cerebral Cortex, 21, 1283-1294.

Schaffert, N., Barrass, K., \& Effenberg, A. O. (2009). Exploring function and aesthetics in sonification for elite sports. In R. Dale, D. Burnham, \& C. J. Stevens (Eds.), Human communication science: A compendium (pp. 465-472). Sydney: ARC Research Network in Human Communication Science.

Scheef, L., Boecker, H., Daamen, M., Fehse, U., Landsberg, M. W., Granath, D. O., et al (2009). Multimodal motion processing in area V5/MT: Evidence from an artificial class of audio-visual events. Brain Research, 1252, 94-104.

Schmidt, R. A., Young, D. E., Swinnen, S., \& Shapiro, D. E. (1989). Summary knowledge of results for skill acquisition: Support for the guidance hypothesis. Journal of Experimental Psychology: Learning, Memory, and Cognition, 15, 352-359.

Schmitz, G., Mohammadi, B., Hammer, A., Heldmann, M., Samii, A., Münte, T. F., et al (2013). Observation of sonified movements engages a basal ganglia frontocortical network. BMC Neuroscience, 14-32.

Sigrist, R., Rauter, G., Riener, R., \& Wolf, P. (2013). Augmented visual, auditory, haptic, and multimodal feedback in motor learning: A review. Psychonomics Bulletin \& Review, 20(1), 21-53.

Smyth, M. M., \& Silvers, G. (1987). Functions of vision in the control of handwriting. Acta Psychologica, 65, 47-64.

Sullivan, K. I., Kantak, S. S., \& Burtner, P. A. (2008). Motor learning in children: Feedback effects on skill acquisition. Physical Therapy, 88(6), 720-732.

Teo, C. L., Burdet, E., \& Lim, H. P. (2002). A robotic teacher of chinese handwriting. In Proceedings of 10th haptic interfaces for virtual environment and teleoperator systems (pp. 335-341).

Thoret, E., Aramaki, M., Gondre, C., Kronland-Martinet, R., \& Ystad, S. (2013). Controlling a non linear friction model for evocative sound synthesis applications. In Proceedings of the 16th international conference on digital audio effects (pp. 1-7). Ireland: Maynooth.

Thoret, E., Aramaki, M., Kronland-Martinet, R., Velay, J. L., \& Ystad, S. (2014). From sound to shape: Auditory perception of drawing movements. Journal of Experimental Psychology: Human Perception and Performance, 40, 983-994.

Van Emmerick, R. E. A., \& Newell, K. M. (1989). The relationship between pen-point and joint kinematics in handwriting and drawing. In R. Plamondon, C. Y. Suen, \& M. L. Simner (Eds.), Computer Recognition and Human Production of Handwriting (pp. 231-248). Singapore: World Scientific.

Varni, G., Dubus, G., Oksanen, S., Volpe, G., Fabiani, M., Bresin, R., et al. (2012). Interactive sonification of synchronization of motoric behaviour in social active listening to music with mobile devices. Journal on Multimodal User Interfaces, 5, $157-173$.

Vogt, K., Pirrò, D., Kobenz, I., Höldrich, R., \& Eckel, G. (2009). PhysioSonic - Evaluated movement sonification as auditory feedback in physiotherapy. In Proceedings of the 6th international conference on auditory display (pp. 103-120).

Wulf, G. (2013). Attentional focus and motor learning: A review of 15 years. International Review of Sport and Exercise Psychology, $6(1), 77-104$.

Wulf, G., \& Schmidt, R. A. (1989). The learning o f generalized motor programs: Reducing the relative frequency of knowledge of results enhances memory. Journal of Experimental Psychology: Learning, Memory, and Cognition, 15, 748-757.

Young, W., Rodger, M., \& Craig, C. M. (2013). Perceiving and reenacting spatiotemporal characteristics of walking sounds. Journal of Experimental Psychology: Human Perception and Performance, 39(2), 464-476. 